This is the accepted manuscript made available via CHORUS, the article has been published as:

\title{
Multiphoton Ionization as a clock to Reveal Molecular Dynamics with Intense Short X-ray Free Electron Laser Pulses
}

L. Fang, T. Osipov, B. Murphy, F. Tarantelli, E. Kukk, J. P. Cryan, M. Glownia, P. H. Bucksbaum, R. N. Coffee, M. Chen, C. Buth, and N. Berrah Phys. Rev. Lett. 109, 263001 - Published 26 December 2012 DOI: 10.1103/PhysRevLett.109.263001 


\title{
Multiphoton Ionization as a "Clock" to Reveal Molecular Dynamics with Intense Short X-ray Free Electron Laser Pulses
}

\author{
L. Fang, ${ }^{1, *}$ T. Osipov, ${ }^{1}$ B. Murphy,${ }^{1}$ F. Tarantelli, ${ }^{2}$ E. Kukk,${ }^{3}$ J.P. Cryan,${ }^{4}$ M. \\ Glownia, ${ }^{4}$ P.H. Bucksbaum, ${ }^{4,5}$ R.N. Coffee, ${ }^{5}$ M. Chen,${ }^{6}$ C. Buth, ${ }^{7}$ and N. Berrah ${ }^{1}$ \\ ${ }^{1}$ Physics Department, Western Michigan University, Kalamazoo, MI 49008, USA \\ ${ }^{2}$ Dipartimento di Chimica, Università di Perugia, and ISTM-CNR, 06123 Perugia, Italy \\ ${ }^{3}$ Department of Physics and Astronomy, University of Turku, 20014 Turku, Finland \\ ${ }^{4}$ The PULSE Institute for Ultrafast Energy Science, \\ SLAC National Accelerator Laboratory, Menlo Park, CA 94025, USA \\ ${ }^{5}$ LCLS, Menlo Park, CA 94025, USA \\ ${ }^{6}$ Lawrence Livermore National laboratory, Livermore, CA 94550, USA \\ ${ }^{7}$ Argonne National Laboratory, Argonne, Illinois 60439, USA
}

(Dated: October 23, 2012)

\begin{abstract}
We investigate molecular dynamics of multiple ionization in $\mathrm{N}_{2}$ through multiple core-level photoabsorption and subsequent Auger decay processes induced by intense, short X-ray free electron laser pulses. The timing dynamics of the photoabsorption and dissociation processes is mapped onto the kinetic energy of the fragments. Measurements of the latter allow us to map out the average internuclear separation for every molecular photoionization sequence step and obtain the average time interval between the photoabsorption events. Using multiphoton ionization as a tool of multiple-pulse pump-probe scheme, we demonstrate the modification of the ionization dynamics as we vary the $\mathrm{x}$-ray laser pulse duration.
\end{abstract}

PACS numbers: 32.30.Rj, 82.80.Ej, 33.20.Rm, 33.60.+q

Substantial efforts have been made both experimentally and theoretically to explore molecular dynamics during and after the interaction of the molecule with radiation, i.e., probe the electrons and nuclei behavior subsequent to absorption of photons [1-4]. Among the number of factors determining this dynamics, the wavelength and intensity of the radiation are particularly important. Recent development of free electron lasers (FELs) has provided a novel source of short X-ray pulses with much higher intensity than available before [5]. However, first experiments on molecular dynamics in this, hitherto unavailable range of parameters, have revealed that our understanding of the involved physics and chemistry is largely qualitative. We are lacking accurate models for quantitatively predicting the behavior of molecular systems that are subjected to intense X-rays interacting directly with atomic inner shells. The motivation of the present study is to remedy this situation by investigating molecular dynamics and fragmentation, subsequent to core ionization and core-hole relaxation, and by providing a model and quantitative description of the interaction. These studies are of fundamental importance to molecular physics, physical chemistry and solid state physics. Furthermore, we expect that, with the rapid development of the FEL sources around the world, the need and the ability to accurately model the early phase of the molecular target breakup (that inevitably follows the intense X-ray pulse) becomes an essential part of many studies.

The interaction of the intense X-ray FEL pulse with a molecule starts in the same way as for a lower inten- sity X-rays, i.e., with single-photon absorption and the ejection of a core electron. In lighter elements such as nitrogen the resulting core hole is filled by Auger decay in a few femtoseconds ( $6.4 \mathrm{fs}$ for $\mathrm{N}_{2}$ ), with the ejection of a second electron (Auger electron). We will refer to this sequence as the photoionization-Auger (PA) cycle. Using conventional X-ray sources, the likelihood of the target molecule absorbing another photon before the dissociation is complete is negligible. In contrast, the photon flux in the FEL pulse is so high that the dissociating molecule has a chance to absorb several X-ray photons in sequence, so that multiple PA cycles (a PAPA... sequence) can take place in the molecular system within just one X-ray FEL pulse [6-10].

In this work, we present an alternative to conventional pump-probe schemes [2, 11-13], taking advantage of the femtosecond timescale of the multiple PA cycles in intense FEL pulses. With suitably chosen pulse intensity, the average interval between the PA cycles can be matched to the dissociation timescale, so that the observer follows the progress of the molecule along the repulsive potential energy surfaces (see Fig. 1). We can regard the multiple PA cycles as an "intrinsic" novel multiple-pulse pump-probe mechanism where each subsequent cycle probes the development of the dynamics. Both the pumping and multiple probing come from a single X-ray pulse by sequential absorption. In the described multiphoton scheme, applicable to any ionization with intense X-ray-FEL pulses, the pump-probe delays are controlled by varying the FEL parameters.

In contrast to the pump-probe schemes based on two 
external pulses, the timing in the present scheme is necessarily of statistical nature, since it is determined by intrinsic quantum events. At each PA cycle, the kinetic energy (KE) of the emitted electrons reflects the molecular potential curves of the relevant charge states at a particular internuclear separation (Fig. 1). The eventual kinetic energy release (KER) to the fragment ions, on the other hand, is determined by the entire dissociation dynamics. By combining these various observables with quantitative modeling, one can extract the averaged timing information and reconstruct molecular dynamics.

Thus, on one hand the PA processes provide a means for studying the electronic and structural dynamics in the X-ray-molecule interaction. And on the other hand it is also an atomic intrinsic tool of quantum control of the interaction, since by manipulating the X-ray beam temporal profile one can modify the dynamics of sequential multiple ionization, opening or closing PA pathways to select desired outcomes $[8,10]$.

In the present study, we examine the measured multiple ionization sequences of $\mathrm{N}_{2}$ molecules with X-ray FEL pulses at equal pulse energies and various pulse durations, which result in different dynamics profiles of the interaction. We model the scenario of the interaction and quantitatively map the time dependence onto the average $\mathrm{KE}$ of the fragment ions. We extract the average time interval between photoabsorption events $\Delta t_{\text {avg }}$ as well as the corresponding internuclear separation $(R)$ at which each step occurs in an ionization sequence. As expected, we observe that $\Delta t_{\text {avg }}$ decreases with increasing intensity: a) at equal pulse energy which leads to a certain final charge state, $\Delta t_{\text {avg }}$ decreases with decreasing pulse duration, e.g., $\Delta t_{\text {avg }}$ decreases from 11-23 fs to 7-12 fs as the pulse duration decreases from 280 fs to 80 fs; b) at equal pulse duration, $\Delta t_{\text {avg }}$ decreases with increasing pulse energy, e.g., $\Delta t_{\text {avg }}$ decreases from 12-23 fs to 7-11 fs in X-ray spatial regions with increasing pulse energies where $\mathrm{N}^{3+}$ to $\mathrm{N}^{7+}$ are preferentially produced. This work takes advantage of sequential PA processes to reveal the dynamics timing by developing a model, which is validated by the measurement of the fragment charge states. It shows the ability to quantify the correlation between ionization rates and KERs, and the possibility to resolve individual photoabsorption step in multiphoton ionizations. Also, this work presents the modification of $\Delta t_{\text {avg }}$ with manipulation of the X-ray pulse duration and shows the variation of the ionization timing dynamics due to different pulse intensities within a single pulse.

In the current experiment, the first PA step produces molecular dications $\mathrm{N}_{2}^{2+}$ in either the ground state or excited states, with vacancies in outer molecular orbitals. About $80 \%$ of the electronic states of the dications that are formed in the Frank-Condon region, are dissociative $[14,15]$. Throughout this work, we will refer to the dissociation process $\mathrm{N}_{2}^{n+m} \rightarrow \mathrm{N}^{n+}+\mathrm{N}^{m+}$ as the (n,m) channel. While the molecule breaks up, other PA cycles can take place locally at each of the atoms and thus the dissociation process may jump from the $(1,1)$ to the $(3,1)$ and then to the $(3,3)$ channel. For a stable molecular dication the dissociation can be started in the $(2,2)$ channel following the second PA process, then jumps to $(4,2)$ and $(4,4)$ channels by subsequent PA's. Similarly, higher charge states can be populated with further PA steps. The ionization sequences can be primarily defined by the final charge of the atomic fragments, but the sequences have common intermediate ionization steps. (See summary of possible pathways in Ref. [16]).

Starting with the ground state of neutral $\mathrm{N}_{2}$, each photoionization step will lift the newly produced molecular ion to a potential curve of a higher charge state, as illustrated schematically in Fig. 1. Each transition occurs at a different time and, once the dissociation starts, at a different $R$. For an ionization sequence, $\Delta t_{\text {avg }}$ is determined by the inverse rate of the core ionization, which is given by the product of the core ionization cross-section and the X-ray flux. Hence, the higher the X-ray-FEL intensity, the shorter $\Delta t_{\text {avg }}$. This gives dissociation less time to proceed between ionizations and hence corresponds to a smaller $R$ for each PA step. The smaller the $R$ at which an ionization occurs, the stronger the influence of the neighboring atom on the one that hosts the photoionization process.



FIG. 1: (Color online) Schematic of the PA sequences while molecules dissociate. The PA sequence shown here as an example is $\mathrm{N}_{2} \rightarrow(1,1) \rightarrow(3,1) \rightarrow(3,3)$. "P": photoionization; "A": Auger decay.

The experiment was performed in the high field physics (HFP) end station of atomic molecular and optical physics (AMO) hutch at the Linac Coherent Light Source (LCLS) [8, 17-19]. The photon energy used was 1100 $( \pm 15) \mathrm{eV}$, which is above the K-edge of neutral $\mathrm{N}$ and all charge states of $\mathrm{N}_{2}$. The nominal pulse energy values from the gas detector monitors are measured upstream of 
the HFP chamber. In the interaction region the actual pulse energy is reduced by $65 \%-85 \%$ due to the beam transport loss $[6,8,17]$. The X-ray beam is focused by a pair of Kirkpatrick-Baez (KB) mirrors to an oval spatial profile with a major axis of $2.2 \mu \mathrm{m}$ and a minor axis of $1.2 \mu \mathrm{m}[6,8]$. The pulse durations used were $280 \mathrm{fs}$, $110 \mathrm{fs}, 80 \mathrm{fs}$ and $7 \mathrm{fs}$, estimated by measuring the width of the electron bunch [20-22]. The data shown in this work were recorded by an ion time-of-flight (iTOF) spectrometer, which is oriented perpendicularly to the plane defined by the polarization and propagation direction of the X-ray beam $[8,9,19]$.

To extract the KE of the ion fragments, we use a SIMION simulation to obtain a basis set of simulated iTOF spectral profiles corresponding to different charge states and KEs [16, 23]. We adjust the weights of the basis for the best fit of the experimental spectra. These iTOF basis functions are generated in such a way that they automatically take into account all the non-trivial spectrometer transmission coefficients for different charge states and $\mathrm{KE}$ values. The average KEs obtained by this method give consistent results when using different basis sets and pools of initial values for fitting to the experimental spectra.

Knowing all the potential energy curves involved in a certain dissociation pathway with concurrent PA sequences, and $\Delta t_{\text {avg }}$, one can calculate the average KER in a channel $(m, n)$. One also needs to know the time profile of the intensity of the FEL pulse; in this work we have approximated it by a square function [22] in which case the ionization rate is constant. The calculation can be performed also in reverse, obtaining KERs from experimentally determined KEs and then extracting R's and times for each preceding PA step. In this calculation we: i) assume the dominant contribution to the observed $\mathrm{N}^{m+}$ ions is from the $(\mathrm{m}, \mathrm{m})$ channel, so that the KER is double that of the measured KE; ii) assume that there is one dominant pathway leading to ions of a certain charge; iii) assume, in the $R$ range of the dissociation of core-ionized molecules, core-hole potential curves have the same slopes as the potential curves of valencehole states populated by the corresponding subsequent Auger decay process, i.e., assume that the Auger decay is instantaneous; iv) set $\Delta t_{\text {avg }}$ inversely proportional to the cross section of the atomic transitions; v) approximate potentials of high charge states with Coulomb potentials (a screening factor is applied to the $(2,2)$ state). The validity of these assumptions and approximations is discussed in the supplementary material [16].

With intense X-ray pulses, we observe highly charged nitrogen fragment ions as a result of PA sequences, as shown in the main panel of Fig. 2. The spectra in Fig. 2 are measured with different pulse durations at equal fluence, except for the $7 \mathrm{fs}$ pulse duration data, for which the pulse energy is lower by $84 \%$. The $7 \mathrm{fs}$ spectrum, from which the PA sequences are essentially missing and which is dominated by processes of molecular nature, is shown as a reference (a case where the least molecular dissociation is involved). As seen in the main panel of Fig. 2, higher X-ray flux results in higher charge states. This may suggest a molecular component to the $R$ dependent atomic core-electron absorption cross section which is changing during molecular dissociation. Also, the dynamics leading to the production of bare $\mathrm{N}^{7+}$ appears to saturate. We observe that the pulse duration has a strong effect not only on the charge state branching ratios [8], but also on the $\mathrm{KE}$ of each ion species. In the inset of Fig. 2, a wider structure corresponds to a larger average KE. For $\mathrm{N}^{+}$and $\mathrm{N}^{2+}$, the peak widths are the same at the three different pulse durations, but starting from $\mathrm{N}^{3+}$ the peak width decreases with increasing pulse duration. To quantify this trend, we obtain the average KE for different ion fragments at different pulse durations, which is shown in Fig. 3. Since molecular ions or atomic fragments are detected after all ionization processes are over, the final KEs are the sum of contributions from all ionization steps involved. The KER shared by the ion fragments is determined by the $R$ where the PA or photoionization processes occur: the smaller the $R$, the larger the resultant KER. In other words, higher charges created at shorter separations convert more repulsive potential energy into the kinetic energy of the ions. As expected and seen in Fig. 3, for $\mathrm{N}^{+}$and $\mathrm{N}^{2+}$, the KEs are independent of the pulse duration, since these ions are mainly formed by PA processes in bound molecular states and the dissociation is not perturbed by further dissociation. For ionic fragments with charges $\geq 3+$ the KE is larger for pulses with shorter duration but with similar fluence, i.e., a higher intensity (see data with pulse durations $\geq 80 \mathrm{fs}$ ). The observed increase of ion $\mathrm{KE}$ for shorter duration pulses supports the reasoning that higher photon intensity reduces $\Delta t_{\text {avg }}$. It is also seen from Fig. 3 that, for a given pulse duration the KE increases with the ion charge. Even at high charge states, e.g., for $\mathrm{N}^{7+}$, we see an increase of $\mathrm{KE}$ compared with that of $\mathrm{N}^{6+}$, reflecting the long range operation of Coulomb repulsion, and showing that the dissociation is not complete after $\mathrm{N}^{5+}$ ionization.

As described previously, with the measured KE and using the above model, we extract $\Delta t_{a v g}$ and the corresponding $R$ for various ionization sequences. This is done independently for different final charge states and the results are shown in Fig. 4. In this figure, each block represents a pathway leading to the corresponding charge state; slices (or dots) of different colors within each block represent the ionization sequences for three different pulse durations. In our model, the time of the first ionization event is based on the average ionization rate, not the start of the FEL pulse, so the absolute starting times shown in Fig. 4 are not equal. For a given final state, the ionization step occurs earlier with a shorter pulse than with a longer pulse. For instance, the $R$ (and 


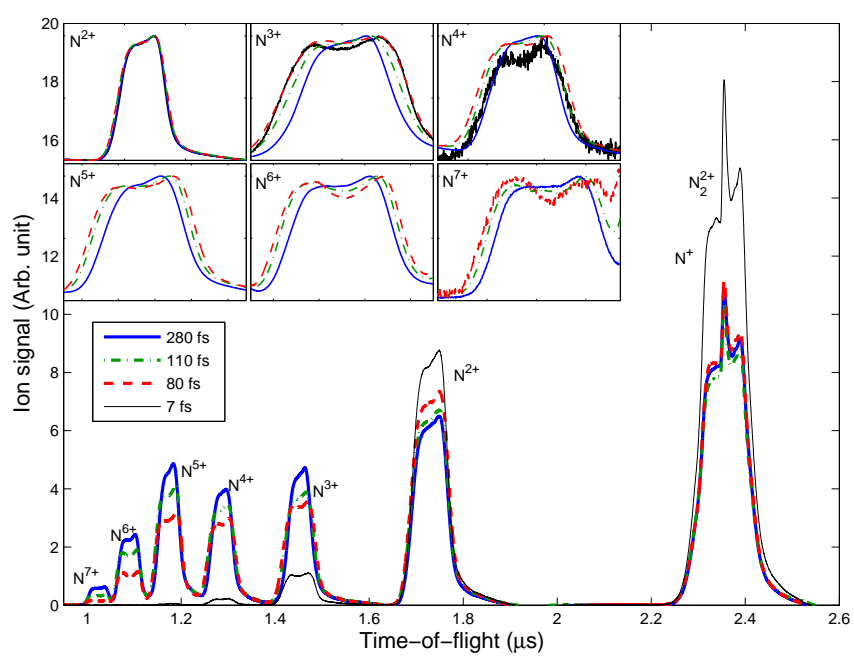

FIG. 2: (Color on line) Ion spectra at different pulse durations. The pulse energy is $1.6-1.9 \mathrm{~mJ}$ (nominal values) for pulse durations $\geq 80 \mathrm{fs}$. The pulse energy for the $7 \mathrm{fs}$ pulse is $0.2-0.3 \mathrm{~mJ}$ (nominal values). Main panel: spectra are normalized to the total ion signal of all charge states. Insets: spectra for different charge states are normalized to the maximum of each spectral peak.

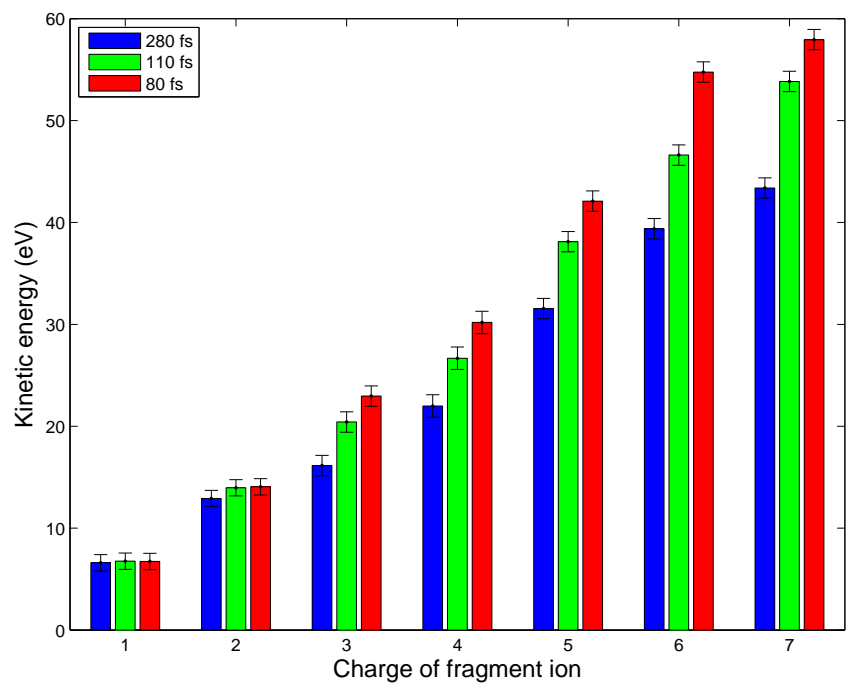

FIG. 3: (Color on line) Average KE of ion fragments at different pulse durations, obtained from the spectra in Fig. 2.

$\left.\Delta t_{\text {avg }}\right)$ to reach $\mathrm{N}^{3+}$ with $80 \mathrm{fs}, 110 \mathrm{fs}$ and $280 \mathrm{fs}$ are $1.73 \pm 0.09 \AA$ (12 fs ), $1.90 \pm 0.15 \AA(15 \mathrm{fs})$ and $2.37 \pm 0.20$ $\AA(23 \mathrm{fs})$. We also find that, for a given pulse duration, the time to reach a specific intermediate charge state is different for ionization sequences leading to different final charge states. For example, using the $\mathrm{KE}$ of $\mathrm{N}^{3+}$, $\mathrm{N}^{5+}, \mathrm{N}^{6+}$ and $\mathrm{N}^{7+}$ at $280 \mathrm{fs}, R$ (and $\Delta t_{\text {avg }}$ ) for the transition $(1,1) \rightarrow(3,1)$ are found to be $2.37 \pm 0.20 \AA(23 \mathrm{fs})$, $1.87 \pm 0.15 \AA(14 \mathrm{fs}), 1.73 \pm 0.09 \AA(12 \mathrm{fs})$ and $1.69 \pm 0.08$ $\AA$ (11 fs), respectively. We thus see a decreasing $R$ and $\Delta t_{\text {avg }}$ for a given intermediate transition in ionization se- quences associated with increasing final ion charges, as they are produced preferentially at increasing energies within a single pulse (see inset of Fig. 4).

The spatial intensity distribution in the focus leads to regions of high and low ionization rates and the measured KE is the convolution of these regions. Our ability to perform "pulse length resolved" spectroscopy of dissociating $\mathrm{N}_{2}$ molecules reveals that the involved molecular dynamics are influenced by both the spatial and temporal profile of the FEL pulse. Furthermore, the accuracy of the determined timing and $R$ for ionization steps depends on the potential energy curves used in the model. One could retrieve the information of the potential energy curves through measurements of the timing and $R$ which could be achieved in a standard pump-probe experiment.

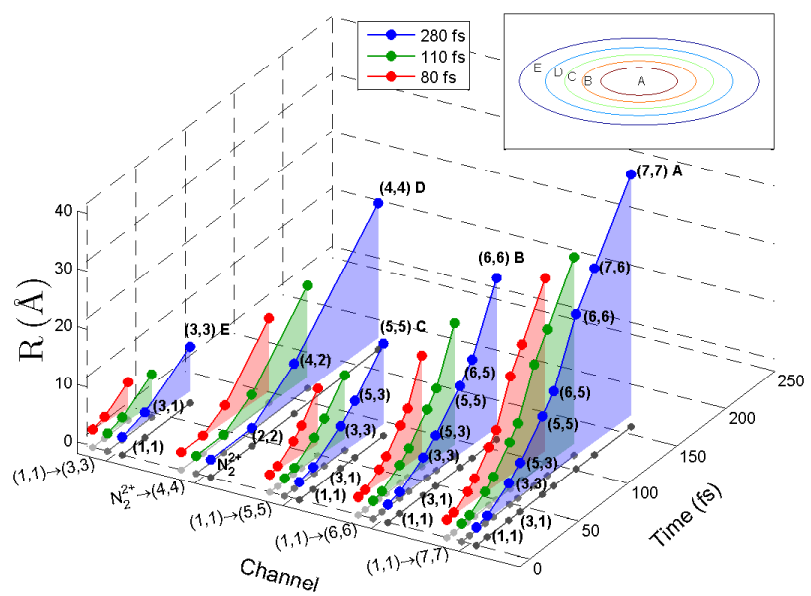

FIG. 4: (Color on line) $R$ as a function of time for different PA pathways at different pulse durations. Each colored dot represents a photoabsorption event. The gray dots represent the projection of the event onto the channel-time plane. The channel axis labels indicate the states after the first PA sequence and the final states. The $\mathrm{N}_{2}^{2+}$ potential curve used in the calculation is the ${ }^{1} \Delta_{g}$ state from Ref. [24]. Inset: schematic of the spatial intensity profile of the FEL beam; the labels correspond to the letter labels in the main panel, indicating that higher charge-state ions are preferentially produced in the region with higher pulse intensity.

In summary, we investigate the ionization dynamics of $\mathrm{N}_{2}$ molecules through sequential multiphoton core-level ionization and subsequent Auger decay. Qualitatively, we expect and observe the molecular dynamics varying as a function of the X-ray intensity. With ion modeling, we are able to quantify the correlations between pulse durations, photoabsorption rates and fragment KEs. We obtain the average ion KE versus charge state and extract $\Delta t_{\text {avg }}$ and the corresponding $R$. With the multiphoton ionization scheme, we demonstrate the modification of the dynamics by varying the X-ray temporal profile. To completely disentangle the photoionization and the fol- 
lowing Auger decay and further investigate the molecular dynamics, an in-depth modeling involving photoabsorption cross sections, Auger decay rates and molecular potential surfaces are necessary. Pump-probe experiments with X-ray pulses much shorter than the Auger decay time may in the future provide effective control of the involved dynamics, while also serving as a powerful FEL beam diagnostics tool. Finally, this work presents a quite general approach for treating ionization sequences accompanied by break-up of gas phase species, in cases of very high photon densities common at FEL sources.

\section{ACKNOWLEDGMENTS}

We thank B. McFarland, C. Blaga, C. Bostedt, D. Rolles, E. P. Kanter, E. Hosler, J. D. Bozek, L. DiMauro, M. Güehr, M. Hoener, M. Messerschmidt, O. Gessner, O. Kornilov, S.R. Leone, S.T. Pratt and V. Petrovic for their earlier contribution. We thank C. L. Cocke and S.T. Pratt for their comments on the manuscript. This work was supported by the U.S. Department of Energy, Office of Science, Basic Energy Sciences, Division of Chemical Sciences, Geosciences, and Biosciences. E.K. acknowledges financial support from the Academy of Finland. C.B. was supported by the Division of Chemical Sciences, Geosciences, and Biosciences of the Office of Basic Energy Sciences, Office of Science, U.S. Department of Energy, under Contract No. DE-AC02-06CH11357. The LCLS is funded by DOE-BES. We thank the LCLS staff for their assistance.

* Electronic address: lifang@slac.stanford.edu
[1] H. J. Wörner and J. B. Bertrand and P. B. Corkum and D. M. Villeneuve, Phys. Rev. Lett. 105, 103002 (2010).

[2] R. Torres, et al., Phys. Rev. A 81, 051802 (2010).

[3] M. J. J. Vrakking, D. M. Villeneuve, and A. Stolow, Phys. Rev. A 54, R37 (1996).

[4] L. J. Frasinski, K. Codling, P. Hatherly, J. Barr, I. N. Ross, and W. T. Toner, Phys. Rev. Lett. 58, 2424 (1987).

[5] J. Amann, et al., Nature Photonics 6, 693 (2012).

[6] L. Fang et al., Phys. Rev. Lett. 105, 083005 (2010).

[7] N. Berrah et al., PNAS 41, 16912 (2011).

[8] M. Hoener et al., Phys. Rev. Lett. 104, 253002 (2010).

[9] J.P. Cryan et al., Phys. Rev. Lett. 105, 083004 (2010).

[10] C. Buth, et al., J. Chem. Phys 136, 214310 (2012).

[11] G. Marcus, et al., Phys. Rev. Lett. 108, 023201 (2012).

[12] L. Fang and G. N. Gibson, Phys. Rev. A 81, 033410 (2010) (and references therein).

[13] A. Assion, M. Geisler, J. Helbing, V. Seyfried, and T. Baumert, Phys. Rev. A 54, R4605 (1996) (and references therein).

[14] H. Ågren, J. Chem. Phys 75, 1267 (1981).

[15] J.P. Cryan et al., J. Phys. B 45, 055601 (2012).

[16] Supplemental materials.

[17] L. Young et al., Nature 466, 56 (2010).

[18] P. Emma et al., Phys. Rev. Lett. 92, 074801 (2004).

[19] J. D. Bozek et al., Eur. Phys. J. Special Topics 169, 129 (2001)

[20] K. L. F. Bane et al., Phys. Rev. Spec. Top. Accel. Beams 12, 030704 (2009).

[21] Y. Ding et al., Phys. Rev. Lett. 102, 254801 (2009).

[22] S. Düsterer et al., New Journal of Physics 13, 093024 (2011).

[23] O. Gessner, et al., Ultrafast Phenomena XVII, M. Chergui, D. M. Jonas, E. Riedle, R. W. Schoenlein, A. J. Taylor, Eds.,Oxford University Press, 47 (2011).

[24] R. W. Wetmore and R. K. Boyd, J. Phys. Chem. 90, 5540 (1986). 